International Journal of Pure and Applied Mathematics

Volume 105 No. 3 2015, 399-413

ISSN: 1311-8080 (printed version); ISSN: 1314-3395 (on-line version)

url: http://www.ijpam.eu

doi: http://dx.doi.org/10.12732/ijpam.v105i3.8

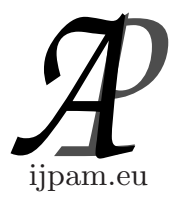

\title{
ON THE EXPONENTIAL CHEBYSHEV APPROXIMATION IN UNBOUNDED DOMAINS: A COMPARISON STUDY FOR SOLVING HIGH-ORDER ORDINARY DIFFERENTIAL EQUATIONS
}

\author{
M.A. Ramadan ${ }^{1}$, K.R. Raslan ${ }^{2}$, T.S. El Danaf ${ }^{3}$, M.A. Abd El Salam ${ }^{4}$ \\ ${ }^{1,3}$ Mathematics Department \\ Faculty of Science \\ Menoufia University \\ Shebein El-Koom, EGYPT \\ ${ }^{2,4}$ Mathematics Department \\ Faculty of Science \\ Al-Azhar University \\ Nasr-City,11884, Cairo, EGYPT
}

\begin{abstract}
In this paper we investigate an improved scheme for exponential Chebyshev (EC) collocation method. The improved scheme of the EC functions is derived and introduced for solving high-order linear ordinary differential equations with variable coefficients in unbounded domain. This technique transforms the given differential equation and mixed conditions to matrix equation with unknown EC coefficients. These matrices together with the collocation method are utilized to reduce the solution of higher-order ordinary differential equations to the solution of a system of algebraic equations. The solution is obtained in terms of EC functions. Numerical examples are given to demonstrate the validity and applicability of the method. The obtained numerical results are compared with others existing methods and the exact solution where it shown to be very attractive with good accuracy.
\end{abstract}

Received: August 16, 2015

(c) 2015 Academic Publications, Ltd. url: www.acadpubl.eu

${ }^{\S}$ Correspondence author 
AMS Subject Classification: 65L20, 65L05, 65L10

Key Words: exponential Chebyshev functions, higher-order ordinary differential equations, exponential Chebyshev collocation method

\section{Introduction}

Chebyshev polynomials are one of the most important special functions, which are widely used in numerical analysis. The well-known Chebyshev first kind polynomials $T_{n}(x)$ [5] are orthogonal with respect to the weight-function $w(x)=$ $1 / \sqrt{1-x^{2}}$ on the interval $[-1,1]$ and the recurrence relation is

$$
T_{0}(x)=1, T_{1}(x)=x, T_{n+1}(x)=2 x T_{n}(x)-T_{n-1}(x), n \geq 1
$$

One of the applications of Chebyshev polynomials is the solution of ordinary differential equations with boundary conditions. Many studies are considered on the interval $[-1,1]$ in which Chebyshev polynomials are defined. Therefore, this limitation causes a failure of the Chebyshev approach in the problems that are naturally defined on larger domains, especially including infinity. Under a transformation that maps the interval $[-1,1]$ into a semi-infinite domain $[0, \infty)$, Boyd [2],[3], Parand et al. [6],[7], and Sezer [9],[10] successfully applied spectral methods to solve problems on semi-infinite intervals. In their studies the basis functions called rational Chebyshev functions, and defined by

$$
R_{n}(x)=T_{n}\left(\frac{x-1}{x+1}\right),
$$

Recently, Koc, and Kurnaz [4] have proposed modified type of Chebyshev polynomials as an alternative to the solutions of the problems given in all real domain. In their studies, the basis functions called exponential Chebyshev functions $E_{n}(x)$ that are orthogonal in $(-\infty, \infty)$. This kind of extension tackles the problems over the whole real domain. Therefore, we introduce a new improved type of exponential Chebyshev functions.

\section{Definition and Properties of Exponential Chebyshev (EC) Functions}

The exponential Chebyshev EC functions are defined by

$$
E_{n}(x)=T_{n}\left(\frac{e^{x}-1}{e^{x}+1}\right),
$$


where the corresponding recurrence relation is

$$
E_{0}(x)=1, E_{1}(x)=\frac{e^{x}-1}{e^{x}+1}, E_{n+1}(x)=2\left(\frac{e^{x}-1}{e^{x}+1}\right) E_{n}(x)-E_{n-1}(x) . n \geq 1
$$

\subsection{Orthogonality of EC Functions}

In the next Proposition we define the form of the weight function needed to guarantee the orthogonality of EC function.

Proposition 1. The weight function $w(x)$ corresponding to EC functions, such that they are orthogonal in the interval $(-\infty, \infty)$ is given by $\sqrt{e^{x}} /\left(e^{x}+1\right)$, with the orthogonal condition

$$
\int_{-\infty}^{\infty} E_{n}(x) E_{m}(x) w(x) d x=\frac{c_{m} \pi}{2} \delta_{n m}
$$

where

$$
c_{m}= \begin{cases}2, & m=0 \\ 1, & m \neq 0\end{cases}
$$

and $\delta_{n m}$ is the Kronecker function [4].

Also the product relation of EC functions used in the derivative relations is given by

$$
E_{n}(x) E_{m}(x)=\frac{1}{2}\left[E_{n+m}(x)+E_{|n-m|}(x)\right] .
$$

\subsection{Function Expansion in Term of EC Functions}

A function $f(x)$ well defined over the interval $(-\infty, \infty)$, can be expanded as

$$
f(x)=\sum_{n=0}^{\infty} a_{n} E_{n}(x),
$$

where

$$
a_{n}=\frac{2}{c_{n} \pi} \int_{-\infty}^{\infty} E_{n}(x) f(x) w(x) d x .
$$

If $f(x)$ in expression (5) is a truncated to $N<\infty$ in terms of the EC functions as

$$
f(x) \cong \sum_{n=0}^{N} a_{n} E_{n}(x)=\mathrm{E}(x) \mathrm{A},
$$


where $\mathbf{E}(x)$ is $1 \times(N+1)$ vector with elements $E_{n}(x)$ and $\boldsymbol{A}$ is an unknown coefficient vector as,

$$
\mathbf{E}(x)=\left[E_{0}(x) E_{1}(x) \ldots E_{N}(x)\right], \quad \boldsymbol{A}=\left[a_{0} a_{1} \ldots a_{N}\right]^{T} .
$$

The $(k)$ th-order derivative of $f(x)$ can be written as

$$
f^{(k)}(x) \cong \sum_{n=0}^{N} a_{n}\left(E_{n}(x)\right)^{(k)}=\mathbf{E}^{(k)}(x) \mathbf{A},
$$

where

$$
\left(E_{n}(x)\right)^{(0)}=E_{n}(x), \quad \mathbf{E}^{(k)}(x)=\left[\left(E_{0}(x)\right)^{(\mathrm{k})}\left(E_{1}(x)\right)^{(\mathrm{k})} \ldots\left(E_{N}(x)\right)^{(\mathrm{k})}\right] .
$$

\subsection{The Derivatives of EC Functions}

Proposition 2. The relation between the row vector $\mathbf{E}(x)$ and its $(k)$ thorder derivative is given as

$$
\boldsymbol{E}^{(k)}(x) \cong \boldsymbol{E}(x)\left(\boldsymbol{D}^{T}\right)^{k},
$$

where, $\boldsymbol{D}$ is the $(N+1) \times(N+1)$ operational matrix for the derivative, and the general form of the matrix $\boldsymbol{D}$ is a tridiagonal matrix which is obtained from

$$
\mathbf{D}=\operatorname{diag}\left(\frac{i}{4}, 0, \frac{-i}{4}\right), \quad i=0,1, \ldots, N .
$$

Proof. Derivatives of the EC functions can be found by differentiating relation (2), and by the help of (4) we get

$$
\begin{gathered}
\left(E_{0}(x)\right)^{\prime}=0, \\
\left(E_{1}(x)\right)^{\prime}=\frac{2 e^{x}}{\left(1+e^{x}\right)^{2}}=\frac{1}{4} E_{0}(x)-\frac{1}{4} E_{2}(x),
\end{gathered}
$$

and

$$
\begin{aligned}
\left(E_{n+1}(x)\right)^{\prime} & =\frac{d}{d x}\left[2 E_{1}(x) E_{n}(x)-E_{n-1}(x)\right] \\
& =\frac{d}{d x}\left[2\left(E_{1}(x)\right)^{(0)}\left(E_{n}(x)\right)^{(0)}-\left(E_{n-1}(x)\right)^{(0)}\right] \\
& =\left[2\left(E_{1}(x)\right)^{(1)}\left(E_{n}(x)\right)^{(0)}+2\left(E_{1}(x)\right)^{(0)}\left(E_{n}(x)\right)^{(1)}-\left(E_{n-1}(x)\right)^{(1)}\right],
\end{aligned}
$$


that can be written as

$$
\left(E_{n+1}(x)\right)^{\prime}=2\left\{\left(E_{1}(x) E_{n}(x)\right)^{\prime}\right\}-\left(E_{n-1}(x)\right)^{\prime} .
$$

By using the relations (10)-(12) and by the help of product relation (4) for $\{n=0,1, \ldots, N\}$, then we get

$$
\left\{\begin{aligned}
\left(E_{0}(x)\right)^{\prime} & =0 \\
\left(E_{1}(x)\right)^{\prime} & =\frac{1}{4} E_{0}(x)-\frac{1}{4} E_{2}(x), \\
\left(E_{2}(x)\right)^{\prime} & =\frac{1}{2} E_{1}(x)-\frac{1}{2} E_{3}(x), \\
& \vdots \\
\left(E a_{n}(x)\right)^{\prime} & =\frac{n}{4} E_{n-1}(x)-\frac{n}{4} E_{n+1}(x), \quad n>1,
\end{aligned}\right.
$$

the previous equalities form $(N+1) \times(N+2)$ matrix then we make a truncation to the last column to get square operational matrix $\mathbf{D}$ given in (10), then to obtain the matrix $\mathbf{E}^{(k)}(x)$ we can use the relations (14) as

$$
\begin{aligned}
\mathbf{E}^{\prime}(x) & \cong \mathbf{E}(x) \mathbf{D}^{\mathrm{T}}, \\
\mathbf{E}^{\prime \prime}(x) & \cong \mathbf{E}^{\prime}(x) \mathbf{D}^{\mathrm{T}}=\left(\mathbf{E}(x) \mathbf{D}^{\mathrm{T}}\right) \mathbf{D}^{\mathrm{T}}=\mathbf{E}(x)\left(\mathbf{D}^{\mathrm{T}}\right)^{2}, \\
\mathbf{E}^{(3)}(x) & \cong \mathbf{E}^{\prime \prime}(x) \mathbf{D}^{\mathrm{T}}=\mathbf{E}(x)\left(\mathbf{D}^{\mathrm{T}}\right)^{3},
\end{aligned}
$$

then we can write

$$
\mathbf{E}^{(k)}(x) \cong \mathbf{E}(x)\left(\mathbf{D}^{T}\right)^{k} .
$$

Proposition 2 and its proof are derived a regular scheme for the relation between the vector $\mathbf{E}(x)$ and its $(k)$ th-order derivative, a similar proof found in [4] with less details. Now we turn to introduce a new improved scheme of the vector $\mathbf{E}(x)$ and its $(k)$ th-order derivative that leads us to get equality sign in (15), as explained in next proposition

Proposition 3. The derivatives of the vector

$$
\boldsymbol{E}(x)=\left[E_{0}(x) E_{1}(x) \ldots . E_{N}(x)\right],
$$

can be expressed with equality sign by

$$
\boldsymbol{E}^{\prime}(x)=\boldsymbol{E}(x) \boldsymbol{D}^{T}+\boldsymbol{B}(x),
$$


where $\boldsymbol{D}$ is $(N+1) \times(N+1)$ operational matrix for the derivative given in (10), and $\boldsymbol{B}(x)$ is $1 \times(N+1)$ row vector which is an actual term to get the equality sign of (16), that was truncated in (9). This added term will improve the obtained approximate solutions as will be shown in the numerical examples in section 6 . And $\boldsymbol{B}(x)$ is deduced as shown below:

$$
\boldsymbol{B}(x)=\left[00 \ldots 0 \frac{-N}{4} E_{N+1}\right] .
$$

Consequently, to obtain the matrix $\mathbf{E}^{(k)}(x)$, we can use the relation (16) as

$$
\begin{aligned}
& \mathbf{E}^{\prime}(x)=\mathbf{E}(x) \mathbf{D}^{T}+\mathbf{B}(x), \\
& (\mathbf{E}(x))^{(2)}=\mathbf{E}^{\prime}(x) \mathbf{D}^{T}+\mathbf{B}^{\prime}(x)=\left(\mathbf{E}(x) \mathbf{D}^{T}+\mathbf{B}(x)\right) \mathbf{D}^{T}+\mathbf{B}^{\prime}(x),
\end{aligned}
$$

then

$$
\mathbf{E}^{(k)}(x)=\mathbf{E}(x)\left(\mathbf{D}^{T}\right)^{k}+\sum_{i=0}^{k-1} \mathbf{B}^{(i)}(x)\left(\mathbf{D}^{T}\right)^{k-i-1}, \quad k \geq 1
$$

where

$$
\mathbf{B}^{(i)}(x)=\left[\begin{array}{lllll}
0 & 0 & \cdots & 0 & \frac{-N}{4} E_{N+1}^{(i)}(x)
\end{array}\right] .
$$

For example at $N=4$ the two matrices $\mathbf{D}$ and $\mathbf{B}(x)$ takes the form

$$
\mathbf{D}=\left[\begin{array}{ccccc}
0 & 0 & 0 & 0 & 0 \\
\frac{1}{4} & 0 & \frac{-1}{4} & 0 & 0 \\
0 & \frac{1}{2} & 0 & \frac{-1}{2} & 0 \\
0 & 0 & \frac{3}{4} & 0 & \frac{-3}{4} \\
0 & 0 & 0 & 1 & 0
\end{array}\right], \quad \mathbf{B}(x)=\left[\begin{array}{lllll}
0 & 0 & 0 & 0 & -E_{5}
\end{array}\right] .
$$

\section{Application of the Introduced two Schemes for Studying Higher-order Ordinary Differential Equations}

The form of high-order linear non-homogeneous differential equations with variable coefficients in unbounded domains is

$$
\sum_{k=0}^{m} P_{k}(x) y^{(k)}(x)=f(x), \quad-\infty<x<\infty,
$$

with the mixed conditions

$$
\sum_{k=0}^{m-1} \sum_{j=0}^{J} d_{i j}^{k} y^{(k)}\left(b_{j}\right)=\alpha_{i},
$$




$$
-\infty<b_{j}<\infty, \quad i=0,1, \ldots, m-1, \quad j=0,1, \ldots, J
$$

where the $P_{k}(x)$ and $f(x)$ are continuous functions on the interval $(-\infty, \infty)$, $d_{i j}^{k}, \quad b_{j}$ and $\alpha_{i}$ are appropriate constants or $b_{j}$ may tends to $\pm \infty$.

Now, we consider that the approximate solution $y_{N}(x)$ to the exact solution $y(x)$ of Eq. (19) defined by expression (6) and its $(k)$ th-order derivative defined by expression (7) as

$$
y_{N}(x)=\sum_{n=0}^{N} a_{n} E_{n}(x)=\mathbf{E}(x) \mathbf{A}
$$

and

$$
y_{N}^{(k)}(x) \cong \sum_{n=0}^{N} a_{n}\left(E_{n}(x)\right)^{(k)}=\mathbf{E}^{(k)}(x) \mathbf{A},
$$

substituting the relation (15) into expression (22), we have the regular scheme of the $(k)$ th-order derivative of the solution function $y_{N}(x)$ of the higher-order differential equations as

$$
\left[y_{N}^{(k)}(x)\right]=\mathbf{E}(x)\left(\mathbf{D}^{\mathrm{T}}\right)^{k} \mathbf{A},
$$

and by substituting the relation (18) into expression (22), we get the improved scheme of the $(k)$ th-order derivative of the solution $y(x)$ as

$$
\left[y_{N}^{(k)}(x)\right]=\left[\mathbf{E}(x)\left(\mathbf{D}^{T}\right)^{k}+\sum_{i=0}^{k-1} \mathbf{B}^{(i)}(x)\left(\mathbf{D}^{T}\right)^{k-i-1}\right] \mathbf{A} .
$$

\section{Fundamental Matrix Relations}

Let us define the collocation points [4], so that $-\infty<x_{i}<\infty$, as

$$
x_{i}=\operatorname{Ln}\left[\frac{1+\cos \left(\frac{i \pi}{N}\right)}{1-\cos \left(\frac{i \pi}{N}\right)}\right], \quad i=1, \ldots, N-1
$$

and at the boundaries $(i=0, \quad i=N) \quad x_{0} \rightarrow \infty, x_{N} \rightarrow-\infty$, since the EC functions are convergent at both boundaries $\pm \infty$, namely their values are \pm 1 , the appearance of infinity in the collocation points does not cause a loss in the method. Then, we substitute the collocation points (25) into Eq. (19) to obtain

$$
\sum_{k=0}^{m} P_{k}\left(x_{i}\right) y_{N}^{(k)}\left(x_{i}\right)=f\left(x_{i}\right), \quad i=0,1, \ldots, N .
$$


The system (26) can be written in the matrix form

$$
\sum_{k=0}^{m} \mathbf{P}_{k} \mathbf{Y}^{(k)}=\mathbf{F}
$$

where

$$
\mathbf{P}_{k}=\left[\begin{array}{cccc}
P_{k}\left(x_{0}\right) & 0 & \ldots & 0 \\
0 & P_{k}\left(x_{1}\right) & \ldots & 0 \\
0 & 0 & \ddots & \vdots \\
0 & 0 & \ldots & P_{k}\left(x_{N}\right)
\end{array}\right], \quad \mathbf{F}=\left[\begin{array}{lllll}
f\left(x_{0}\right) & f\left(x_{1}\right) & \ldots & f\left(x_{N}\right)
\end{array}\right]^{\mathrm{T}} .
$$

By putting the collocation points $x_{i}$ in the regular scheme (23), we have the system

or

$$
\left[y_{N}^{(k)}\left(x_{i}\right)\right]=\mathbf{E}\left(x_{i}\right)\left(\mathbf{D}^{\mathrm{T}}\right)^{k} \mathbf{A}, \quad i=0,1, \ldots, N
$$

$$
\begin{gathered}
\mathbf{Y}^{(k)}=\left[\begin{array}{c}
y_{N}^{(k)}\left(x_{0}\right) \\
y_{N}^{(k)}\left(x_{1}\right) \\
\vdots \\
y_{N}^{(k)}\left(x_{N}\right)
\end{array}\right]=\mathbf{E}\left(\mathbf{D}^{T}\right)^{k} \mathbf{A}, \\
\mathbf{E}=\left[\begin{array}{c}
E\left(x_{0}\right) \\
E\left(x_{1}\right) \\
\vdots \\
E\left(x_{N}\right)
\end{array}\right]=\left[\begin{array}{cccc}
E_{0}\left(x_{0}\right) & E_{1}\left(x_{0}\right) & \ldots & E_{N}\left(x_{0}\right) \\
E_{0}\left(x_{1}\right) & E_{1}\left(x_{1}\right) & \ldots & E_{N}\left(x_{1}\right) \\
\vdots & \vdots & \ddots & \vdots \\
E_{0}\left(x_{N}\right) & E_{1}\left(x_{N}\right) & \ldots & E_{N}\left(x_{N}\right)
\end{array}\right] .
\end{gathered}
$$

And the fundamental matrix will be in the form

$$
\sum_{k=0}^{m} \mathbf{P}_{k} \mathbf{E}(x)\left(\mathbf{D}^{\mathrm{T}}\right)^{k} \mathbf{A}=\mathbf{F} \text {. }
$$

Next the corresponding matrix form for the condition can be written as follows

$$
\begin{gathered}
\sum_{k=0}^{m-1} \sum_{j=0}^{J} d_{i j}^{k} \mathbf{E}\left(b_{j}\right)\left(\mathbf{D}^{\mathrm{T}}\right)^{k} \mathbf{A}=\left[\alpha_{i}\right], \\
-\infty<b_{j}<\infty, \quad i=0,1, \ldots, m-1, \quad j=0,1, \ldots, J
\end{gathered}
$$

Now by using the improved scheme (24) the fundamental matrix is

$$
\sum_{k=0}^{m} \mathbf{P}_{k}\left\{\mathbf{E}\left(\mathbf{D}^{T}\right)^{k}+\sum_{i=0}^{k-1} \mathbf{B}^{(i)}\left(\mathbf{D}^{T}\right)^{k-i-1}\right\} \mathbf{A}=\mathbf{F} .
$$


Next the corresponding matrix form for the condition can be written as follows

$$
\begin{gathered}
\sum_{k=0}^{m-1} \sum_{j=0}^{J} d_{i j}^{k}\left\{\mathbf{E}\left(b_{j}\right)\left(\mathbf{D}^{\mathrm{T}}\right)^{k}+\sum_{\mathrm{i}=0}^{\mathrm{k}-1} \mathbf{B}^{(i)}\left(b_{j}\right)\left(\mathbf{D}^{\mathrm{T}}\right)^{k-i-1}\right\} \mathbf{A}=\left[\alpha_{i}\right], \\
-\infty<b_{j}<\infty, \quad i=0,1, \ldots, m-1, \quad j=0,1, \ldots, J
\end{gathered}
$$

where

$$
\mathbf{B}=\left[\begin{array}{c}
\mathbf{B}\left(x_{0}\right) \\
\mathbf{B}\left(x_{1}\right) \\
\vdots \\
\mathbf{B}\left(x_{N}\right)
\end{array}\right]=\left[\begin{array}{cccc}
B_{0}\left(x_{0}\right) & B_{1}\left(x_{0}\right) & \ldots & B_{N}\left(x_{0}\right) \\
B_{0}\left(x_{1}\right) & B_{1}\left(x_{1}\right) & \ldots & B_{N}\left(x_{1}\right) \\
\vdots & \vdots & \ddots & \vdots \\
B_{0}\left(x_{N}\right) & B_{1}\left(x_{N}\right) & \ldots & B_{N}\left(x_{N}\right)
\end{array}\right]
$$

\section{Method of Solution}

The fundamental matrices (27) and (29) for Eq. (19) corresponding to a system of $(N+1)$ algebraic equations for the $(N+1)$ unknown coefficients $a_{0}, a_{1}, \ldots a_{N}$. We can write Eq. (19) as

$$
\boldsymbol{W A}=\boldsymbol{F} \quad \text { or } \quad[\boldsymbol{W} ; \boldsymbol{F}]
$$

so that, for regular scheme (23)

$$
\boldsymbol{W}=\left(w_{p q}\right)=\sum_{k=0}^{m} \mathbf{P}_{k} \mathbf{E}(x)\left(\mathbf{D}^{\mathrm{T}}\right)^{k}, \quad p, q=0,1, \ldots, N
$$

and, for the improved scheme (24) $\boldsymbol{W}$ is

$$
\boldsymbol{W}=\left(w_{p q}\right)=\sum_{k=0}^{m} \mathbf{P}_{k}\left[\mathbf{E}(x)\left(\mathbf{D}^{\mathrm{T}}\right)^{k}+\sum_{i=0}^{k-1} \mathbf{B}^{(i)}\left(\mathbf{D}^{T}\right)^{k-i-1}\right], \quad p, q=0,1, \ldots, N
$$

we can obtain the matrix form for the mixed conditions by means of (28) and (30) as

$$
\mathbf{U}_{i} \mathbf{A}=\left[\alpha_{i}\right] \quad \text { or } \quad\left[\mathbf{U}_{i} ; \alpha_{i}\right], \quad i=0,1, \ldots m-1
$$

where, for (28)

$$
\mathbf{U}_{i}=\left[\begin{array}{llll}
u_{i 0} & u_{i 1} & \ldots & u_{i N}
\end{array}\right]=\sum_{k=0}^{m-1} \sum_{j=0}^{J} d_{i j}^{k} \mathbf{E}\left(b_{j}\right)\left(\mathbf{D}^{T}\right)^{k} .
$$


and, for (30)

$$
\begin{aligned}
\mathbf{U}_{i}=\left[\begin{array}{llll}
u_{i 0} & u_{i 1} & \ldots & u_{i N}
\end{array}\right] \\
=\sum_{k=0}^{m-1} \sum_{j=0}^{J} d_{i j}^{k}\left\{\mathbf{E}\left(b_{j}\right)\left(\mathbf{D}^{T}\right)^{k}+\sum_{i=0}^{k-1} \mathbf{B}^{(i)}\left(b_{j}\right)\left(\mathbf{D}^{\mathrm{T}}\right)^{k-i-1}\right\} .
\end{aligned}
$$

To obtain the solution of Eq. (19) under the given condition by replacing the rows matrices (32) by the last $m$ rows of the matrix (31) we have required augmented matrix

$$
\left[\mathbf{W}^{*} ; \mathbf{F}^{*}\right]=\left[\begin{array}{cccccc}
w_{00} & w_{01} & \ldots & w_{0 N} & ; & f\left(x_{0}\right) \\
w_{10} & w_{11} & \ldots & w_{1 N} & ; & f\left(x_{1}\right) \\
\ldots & \ldots & \ldots & \ldots & ; & \ldots \\
w_{N-m, 0} & w_{N-m, 1} & \ldots & w_{N-m, N} & ; & f\left(x_{N-m}\right) \\
u_{00} & u_{01} & \ldots & u_{0 N} & ; & \alpha_{0} \\
u_{10} & u_{11} & \ldots & u_{1 N} & ; & \alpha_{1} \\
\ldots & \ldots & \ldots & \ldots & ; & \ldots \\
u_{m-1,0} & u_{m-1,1} & \ldots & u_{m-1, N} & ; & \alpha_{m-1}
\end{array}\right]
$$

or the corresponding matrix equation

$$
\mathbf{W}^{*} \mathbf{A}=\mathbf{F}^{*}
$$

if the $\operatorname{rank}\left(\mathrm{W}^{*}\right)=\operatorname{rank}\left[\mathrm{W}^{*} ; \mathrm{F}^{*}\right]=N+1$ then we can write

$$
\mathbf{A}=\left(\mathbf{W}^{*}\right)^{-1} \mathbf{F}^{*}
$$

Thus, the coefficient $a_{i}, i=0,1, \ldots, N$ are uniquely determined by Eq. (33), $(34)$

\section{Test Examples}

Example 1. Consider the following equation

$$
y^{\prime \prime}-\frac{1}{1+e^{x}} y^{\prime}-\frac{15 e^{2 x}}{\left(1+e^{x}\right)^{2}} y=\frac{e^{2 x}}{\left(1+e^{x}\right)^{6}}, \quad x \in(-\infty, \infty)
$$

and the boundary conditions $y(x) \rightarrow 0$ when $|x| \rightarrow \infty$.

For $N=4$, the collocation points are $x_{0,4} \rightarrow \pm \infty, x_{1}=\operatorname{Ln}(3+2 \sqrt{2})$, $x_{2}=0, \quad x_{3}=\operatorname{Ln}(3-2 \sqrt{2})$. 
Here $\mathbf{P}_{2}$ is the identity matrix, and matrices $\mathbf{P}_{0}, \mathbf{P}_{1}, \mathbf{E}, \mathbf{B}$ are in the form

$$
\begin{aligned}
& \mathbf{P}_{0}=\left[\begin{array}{ccccc}
-15 & 0 & 0 & 0 & 0 \\
0 & \frac{-15}{8}(3+2 \sqrt{2}) & 0 & 0 & 0 \\
0 & 0 & \frac{-15}{4} & 0 & 0 \\
0 & 0 & 0 & \frac{15}{8}(-3+2 \sqrt{2}) & 0 \\
0 & 0 & 0 & 0 & 0
\end{array}\right], \\
& \mathbf{B}=\left[\begin{array}{ccccc}
0 & 0 & 0 & 0 & -1 \\
0 & 0 & 0 & 0 & \frac{1}{\sqrt{2}} \\
0 & 0 & 0 & 0 & 0 \\
0 & 0 & 0 & 0 & \frac{-1}{\sqrt{2}} \\
0 & 0 & 0 & 0 & 1
\end{array}\right] \\
& \mathbf{P}_{1}\left[\begin{array}{ccccc}
0 & 0 & 0 & 0 & 0 \\
0 & \frac{1}{4}(-2+\sqrt{2}) & 0 & 0 & 0 \\
0 & 0 & \frac{-1}{2} & 0 & 0 \\
0 & 0 & 0 & \frac{1}{4}(-2-\sqrt{2}) & 0 \\
0 & 0 & 0 & 0 & -1
\end{array}\right], \\
& \mathbf{E}=\left[\begin{array}{ccccc}
1 & 1 & 1 & 1 & 1 \\
1 & \frac{1}{\sqrt{2}} & 0 & \frac{-1}{\sqrt{2}} & -1 \\
1 & 0 & -1 & 0 & 1 \\
1 & \frac{-1}{\sqrt{2}} 0 & 0 & \frac{1}{\sqrt{2}} & -1 \\
1 & -1 & 1 & 0 & 1
\end{array}\right]
\end{aligned}
$$

and the augmented matrix for the conditions with $N=4$ for $x \rightarrow \infty$ is

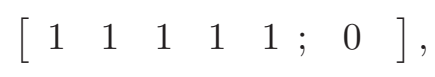

and when $x \rightarrow-\infty$ is

$$
\left[\begin{array}{lllllll}
1 & -1 & 1 & -1 & 1 & ; & 1
\end{array}\right]
$$

then, the fundamental matrix takes the form

$$
\left\{\mathbf{P}_{0} \mathbf{E}\left(\mathbf{D}^{\mathrm{T}}\right)^{0}+\mathbf{P}_{1} \mathbf{E}\left(\mathbf{D}^{\mathrm{T}}\right)^{1}+\mathbf{P}_{2} \mathbf{E}\left(\mathbf{D}^{\mathrm{T}}\right)^{2}\right\} \mathbf{A}=\mathbf{F},
$$

for the regular scheme, and for the improved takes the form

$$
\left\{\mathbf{P}_{0} \mathbf{E}+\mathbf{P}_{1}\left[\mathbf{E}\left(\mathbf{D}^{\mathrm{T}}\right)^{1}+\mathbf{B}\right]+\mathbf{P}_{2}\left[\mathbf{E}\left(\mathbf{D}^{\mathrm{T}}\right)^{2}+\mathbf{B D}^{\mathrm{T}}+\mathbf{B}^{\prime}\right]\right\} \mathbf{A}=\mathbf{F},
$$


After the augmented matrices of the two systems and conditions are computed, we obtain the solutions for the regular scheme,

$$
\mathbf{A}=\left[\begin{array}{lllll}
\frac{2996877+928040 \sqrt{2}}{13222432} & -\frac{1}{2} & \frac{5753473-278412 \sqrt{2}}{26444864} & 0 & \frac{1475205-1577668}{26444864}
\end{array}\right],
$$

and for the improved scheme takes the form

$$
\mathbf{A}=\left[\begin{array}{lllll}
\frac{35}{128} & -\frac{7}{16} & \frac{7}{32} & -\frac{1}{16} & \frac{1}{128}
\end{array}\right]
$$

where,

$$
y_{4}(x)=a_{0} E_{0}+a_{1} E_{1}+a_{2} E_{2}+a_{3} E_{3}+a_{4} E_{4},
$$

then the improved scheme gives for $N=4$

$$
\begin{aligned}
y_{4}(x)= & \frac{35}{128} E_{0}-\frac{7}{16} E_{1}+\frac{7}{32} E_{2}-\frac{1}{16} E_{3}+\frac{1}{128} E_{4} \\
= & \frac{35}{128}-\frac{7}{16}\left(\frac{e^{x}-1}{e^{x}+1}\right)+\frac{7}{32}\left[2\left(\frac{e^{x}-1}{e^{x}+1}\right)^{2}-1\right] \\
& -\frac{1}{16}\left[4\left(\frac{e^{x}-1}{e^{x}+1}\right)^{3}-3\left(\frac{e^{x}-1}{e^{x}+1}\right)\right] \\
& +\frac{1}{128}\left[1+8\left(\frac{e^{x}-1}{e^{x}+1}\right)^{4}-8\left(\frac{e^{x}-1}{e^{x}+1}\right)^{2}\right],
\end{aligned}
$$

that is close to $y_{4}(x)=\frac{1}{\left(1+e^{x}\right)^{6}}$, which represent the exact solution. On the other hand, the regular scheme give us approximate solution for $N=4$, but for $N=6$ we get the exact solution as

$$
\mathbf{A}=\left[\begin{array}{lllllll}
\frac{35}{128} & -\frac{7}{16} & \frac{7}{32} & -\frac{1}{16} & \frac{1}{128} & 0 & 0
\end{array}\right] .
$$

Example 2. Consider the following boundary value problem

$$
L y=f(x), \quad x \in(-\infty, \infty)
$$

where the operator $L=\left[\frac{d^{2}}{d x^{2}}-1\right]$, and the boundary conditions $y(x) \rightarrow 0$ when $|x| \rightarrow \infty$. The exact solution found in [1] by Fourier transform as

$$
y(x)=F^{-1}\left[\frac{-1}{1+\omega^{2}} F[f(x)]\right],
$$

where $F$ and $F^{-1}$ are Fourier and inverse Fourier transform, we apply our present method to Eq. (36), Table. 1 shows the approximate and exact solutions at different $N, x \in[-3,3]$ if we take $f(x)=-2 \operatorname{sech}^{3}(x)$. In Table. 2 
$L_{2}$ and $L_{\infty}$ norms are presented; we can see that for grater $N$ good accuracy is achieved, where the improved scheme has accuracy better than regular scheme. The norms:

$$
\begin{aligned}
L_{2} & =\sqrt{h \sum_{i=0}^{I}\left(y_{\text {Exact }}^{i}-y_{\text {Approximat }}^{i}\right)^{2}}, \\
L_{\infty} & =\text { Max }\left|y_{\text {Exact }}^{i}-y_{\text {Approximat }}^{i}\right| .
\end{aligned}
$$

\begin{tabular}{cccccc}
\hline$x$ & $\begin{array}{c}\text { Exact solution } \\
y(x)=\sec h(x)\end{array}$ & $\begin{array}{c}\text { EC regular } \\
\text { scheme } N=8\end{array}$ & $\begin{array}{c}\text { EC regular } \\
\text { scheme } N=16\end{array}$ & $\begin{array}{c}\text { EC Improved } \\
\text { scheme } N=8\end{array}$ & $\begin{array}{c}\text { EC Improved } \\
\text { scheme } N=16\end{array}$ \\
\hline $\mathbf{- 3 . 0}$ & 0.0993279 & 0.098834 & 0.0993286 & 0.0999047 & 0.0993274 \\
$\mathbf{- 2 . 5}$ & 0.163071 & 0.164516 & 0.163069 & 0.163871 & 0.163071 \\
$\mathbf{- 2 . 0}$ & 0.265802 & 0.268388 & 0.265804 & 0.265956 & 0.265803 \\
$\mathbf{- 1 . 6}$ & 0.387978 & 0.388839 & 0.387978 & 0.386887 & 0.387977 \\
$\mathbf{- 1}$ & 0.648054 & 0.645128 & 0.648056 & 0.64711 & 0.648055 \\
$\mathbf{- 0 . 5}$ & 0.886819 & 0.887489 & 0.886817 & 0.888264 & 0.886818 \\
$\mathbf{0 . 0}$ & 1 & 1.00426 & 1.0 & 1.0018 & 1.0 \\
$\mathbf{0 . 5}$ & 0.886819 & 0.887489 & 0.886817 & 0.888264 & 0.886818 \\
$\mathbf{1}$ & 0.648054 & 0.645128 & 0.648056 & 0.64711 & 0.648055 \\
$\mathbf{1 . 6}$ & 0.387978 & 0.388839 & 0.387978 & 0.386887 & 0.387977 \\
$\mathbf{2 . 0}$ & 0.265802 & 0.268388 & 0.265804 & 0.265956 & 0.265803 \\
$\mathbf{2 . 5}$ & 0.163071 & 0.164516 & 0.163069 & 0.163871 & 0.163071 \\
$\mathbf{3 . 0}$ & 0.0993279 & 0.098834 & 0.0993286 & 0.0999047 & 0.0993274 \\
\hline
\end{tabular}

\begin{tabular}{|c|c|c|c|c|}
\hline & $L_{2}$ regular scheme & $L_{2}$ improved scheme & $L_{\infty}$ regular scheme & $L_{\infty}$ improved scheme \\
\hline$N=8$ & 0.0000850239 & 0.0000231344 & 0.00426021 & 0.00181573 \\
\hline$N=16$ & $4.96036 \times 10^{-11}$ & $6.8930 \times 10^{-12}$ & $2.41935 \times 10^{-6}$ & $1.0313 \times 10^{-6}$ \\
\hline
\end{tabular}

Table 1: Comparing the approximate and exact solution

Table 2: Error norms for Example 2

Example 3. Now we consider the following differential equation

$$
L y=f(x), \quad x \in(-\infty, \infty)
$$

where the operator $L=\left[\frac{d^{3}}{d x^{3}}-1\right]$, and the condition is $y(x) \rightarrow 0$ when $|x| \rightarrow \infty$. The exact solution taken to be same as the previous example i.e. $y(x)=$ $\sec h(x)$, then the function $f(x)$ is $\sec h(x)\left[-1+5 \sec h^{2}(x) \tanh (x)-\tanh ^{3}(x)\right]$, by applying our present method to Eq. (37), Table. 3 shows comparing the $L_{2}$ at different $N$ where $h=0.1$ and $x \in[-3,3]$.

Now, we observe from example three, that the increasing order of differentiation offset by an increase in the truncation in the regular scheme, while the 


\begin{tabular}{|c|c|c|c|c|}
\hline & $L_{2}$ regular scheme & $L_{2}$ improved scheme & $L_{\infty}$ regular scheme & $L_{\infty}$ improved scheme \\
\hline$N=8$ & 0.142527 & 0.00041549 & 0.175465 & 0.0115797 \\
\hline$N=16$ & $3.45619 \times 10^{-7}$ & $2.08173 \times 10^{-11}$ & 0.000454724 & $2.9515 \times 10^{-6}$ \\
\hline
\end{tabular}

Table 3: Error norms for Example 3

improved scheme does not have a truncation, so the values of $L_{2}$ worth almost such as example two.

\section{Conclusion}

In this paper a new improved exponential Chebyshev (EC) collocation method is investigated. The improved method introduced to solve high-order linear ordinary differential equations in unbounded domain. The proposed differential equations and the given conditions were transformed to matrix equation with unknown EC coefficients. This technique is considered to be a modification of the similar presented in $[4],[9],[10]$ and [8]. On the other hand, the EC functions approach deals directly with infinite boundaries without singularities. This variant for our method gave us freedom to solve differential equations with boundary conditions tends to infinity. Illustrative examples are used to demonstrate the applicability and the effectiveness of the proposed technique.

\section{References}

[1] J. Billingham, A.C. King and S.R. Otto, Differential equations linear, nonlinear, ordinary, partial, Cambridge University Press 2003.

[2] J.P. Boyd, Orthogonal rational functions on a semi-infinite interval, $J$. Comput. Phys. 70, 63-88, 1987.

[3] J.P. Boyd, Chebyshev and Fourier spectral methods, Second Edition, DOVER Publications, Mineola, 2000.

[4] A. B. Koc and A. Kurnaz, A new kind of double Chebyshev polynomial approximation on unbounded domains, Boundary Value Problems, 2013: 1, 1687-2770, 2013.

[5] J.C. Mason, D.C. Handscomb, Chebyshev polynomials, CRC Press, Boca Raton, 2003. 
[6] K. Parand and M. Razzaghi, Rational Chebyshev tau method for solving higher- order ordinary differential equations, Inter. J. Comput. Math. 81, 73-80, 2004.

[7] K. Parand and M. Razzaghi, Rational Chebyshev tau method for solving Volterra population model, Applied Mathematics and Computation 149, 893-900, 2004.

[8] M. A. Ramadan, K. R. Raslan and M. A. Nassar, An approximate analytical solution of higher-order linear differential equations with variable coefficients using improved rational Chebyshev collocation method, Applied and Computational Mathematics 3: 6, 315-322, 2014.

[9] M. Sezer, M. Gulsu and B. Tanay, Rational Chebyshev collocation method for solving higher-order linear ordinary differential equations, Wiley Online Library, DOI 10.1002/num.20573, 2010.

[10] S. Yalcinbas, N. Ozsoy and M. Sezer, Approximate solution of higherorder linear differential equations by means of a new rational Chebyshev collocation method, Mathematical and Computational Applications, 15: 1, 45-56, 2010. 
